\title{
IMPORTANCIA Y CULTIVO DE LA UCHUVA (Physalis peruviana L.) ${ }^{1}$
}

\author{
GERHARD FISCHER ${ }^{2}$, PEDRO JOSÉ ALMANZA-MERCHÁN ${ }^{3}$, DIEGO MIRANDA ${ }^{4}$
}

RESUMEN - Physalis perurvianus L., es nativo del Perú y Chile y desde allí ha sido llevado a América Latina, incluyendo Brasil. También fue llevado a la India, Sudáfrica y otros países. Aquí se discute discutir acerca de su importancia económica, aspectos botánicos, su cultivo en varias zonas, plagas, enfermedades y pos-cosecha.

Palabras claves: producción, uchuva, manejo hortícola.

\section{IMPORTÂNCIA E CULTIVO DO (Physalis peruviana L.) NO MUNDO}

RESUMO- Physalis perurvianus L, foi adaptada no Peru e Chile e dai levada para outros países da América Latina, incluindo Brasil além da India, África do Sul e outros. Neste trabalho são discutidas informações sobre seu cultivo em vários países, importancia econômica, aspectos botânicos, propagação, doenças e pragas e pós-colheita.

Termos para indexação: produção, physalis peruviana L., manejo.

\section{INTRODUCCIÓN}

El cultivo de la Uchuva (Physalis peruviana L., Solanaceae) está bien distribuido en las zonas altas de Suramérica y Legge (1974) sitúa su origen en las zonas andinas peruanas. Hay indicios que el fruto llegó de Brasil y fue aclimatado en los altiplanos del Perú y Chile (CRFG, 1997). Se conoce en países como Ecuador bajo el nombre de uvilla, tepareey makowi en la India, chuchuva en Venezuela, aguaymanto en Perú, groselha do Perú en Portugal, Kapstachelbeere en Alemania, Fisalis en Italia, Lampion en Holanda y cape gooseberry (por Ciudad del Cabo) en los países de lengua inglesa (Fischer et al., 2011), mientras el género Physalis proviene del griego "Physa" (vejiga o ampolla) (FISCHER Y MIRANDA, 2012).

La planta que crece inicialmente en forma herbácea, a partir del segundo año forma un arbusto perenne y semileñoso y sus hojas son simples, alternas, acorazonadas y pubescentes con un tamaño entre 5 y $15 \mathrm{~cm}$ de largo y 4 a $10 \mathrm{~cm}$ de ancho (Fischer, 2000). Sin tutorado la planta puede llegar hasta una altura de 1,0 a 1,5 m, ramificándose en forma simpodial y, en muchos casos, genera cuatro ramas reproductivas principales, mientras tutorado puede alcanzar hasta $2 \mathrm{~m}$ o más de altura (figura 1) (FISCHER Y MIRANDA, 2012). La Uchuva presenta un hábito de crecimiento indeterminado, por lo cual el desarrollo de nuevas ramas, hojas y flores ocurre simultáneamente (Fischer et al., 2011).

En las axilas de pedúnculo foliar, se desarrollan las flores hermafroditas, que son solitarias, con una corola tubular de color amarillo (figura 2), y los insectos y el viento las polinizan fácilmente (National Research Council, 1989); sin embargo, la autopolinización es común (Gupta y Roy, 1981).

El cáliz (o capacho) encierra el fruto totalmente hasta su madurez (figura 2) y pierde su clorofila a partir de unos 40-45 días de su desarrollo (Almanza-Merchán y Fischer, 2012), es formado por cinco sépalos persistentes y muestra una estructura similar a una vejiga de unos $5 \mathrm{~cm}$. El cáliz protege el fruto contra insectos, pájaros, patógenos y condiciones climáticas extremas (FISCHER et al., 1997) y además, sirve como empaque natural (GALVIS et al., 2005).

1(Trabalho 441-13). Recebido em: 20-09-2013. Aceito para publicação em: 15-12-2013. Palestra II Simpósio Internacional de Fruticultura- Frutas Exóticas, 21 a 25 de outubro de 2013. Jaboticabal-SP.

${ }^{2}$ Ing. Hort., Ph.D., Departamento de Agronomía, Facultad de Agronomía, Universidad Nacional de Colombia, Bogotá. E-mail: gfischer@unal.edu.co

${ }_{3}^{3}$ Ing. Agr., Ph.D., Facultad de Ciencias Agropecuarias, Universidad Pedagógica y Tecnológica de Colombia, Tunja. E-mail: ppcalma@ gmail.com

${ }^{4}$ Ing. Agr., Ph.D., Departamento de Agronomía, Facultad de Agronomía, Universidad Nacional de Colombia, Bogotá. E-mail: dmirandal@unal.edu.co 
Los frutos de la Uchuva que parecen minitomates, pero de color amarillo-anaranjados, casi redondos, son bayas de 1,25 a 2,5 cm de diámetro y pesan entre unos 4 y $10 \mathrm{~g}$ (Fischer, 1995), contienen entre 150 y 300 semillas aplanadas y de forma lenticular.

El fruto que necesita entre 60 y 80 días para madurar, tiene extraordinarias propiedades nutricionales y medicinales y su exquisito sabor y aroma atraen los consumidores favoreciendo la conquista de nuevos mercados (Galvis et al., 2005), especialmente su alto contenido de provitamina A (1.000-5.000 U.I., principalmente beta-caroteno) y ácido ascórbico (11-42 mg/100 g peso fresco [PF]), $\mathrm{y}$ algunas vitaminas del complejo $\mathrm{B}$ (tiamina, niacina y vitamina $\mathrm{B} 12)$; además se destaca por sus altos contenidos de proteína cruda (2,2 g), fósforo (39 mg) y hierro $(1,1 \mathrm{mg})$, pero con un bajo nivel de calcio (14 mg/100 g PF)(REHM Y ESPIG, 1991; FISCHER Y MIRANDA, 2012). Herrera (2000) encontró entre 13 y $15^{\circ}$ Brix y un buen contenido de ácidos (1,6 a $2,0 \%$ de acidez). Además, el fruto se destaca por su alto contenido de antioxidantes, ácidos grasos poliinsaturados y fitoesteroles (Puente et al., 2011). No obstante, la Uchuva tiene muchos beneficios, el nivel de consumo es muy bajo, por ejemplo en Colombia, los autores estiman que el consumo per cápita es menos de $200 \mathrm{~g}$ por año.

\section{IMPORTANCIA DEL CULTIVO DE LA UCHUVA EN EL MUNDO}

En la actualidad, en el continente americano, el cultivo de la uchuva ha extendido su producción tanto a los altiplanos de los países tropicales y subtropicales, como a países del Caribe (Fischer y Miranda, 2012). Actualmente, en Sudamérica, países como Ecuador, Perú, Chile y Brasil aumentan su área de cultivo.

El cultivo de Uchuva, es una alternativa de producción para la economía de muchos países, debido a que presenta buenas perspectivas e interés en los mercados internacionales, lo cual se deriva de las características nutricionales y propiedades medicinales que posee el fruto (GASTELUM, 2012). Los principales productores de Physalis a nivel mundial: son Colombia, Kenia, Zimbabwe, Australia, Nueva Zelanda, India y Ecuador (BELTRAN, 2009). Se cultiva en menor importancia en: Estados Unidos, Brasil, Venezuela, Bolivia, Perú, Chile, Centroamérica, México, Belice, Guatemala, El Salvador, Nicaragua, Costa Rica, Panamá, Jamaica, África, Sudáfrica, Gabón, Egipto, Asia, India, China, Japón, Malasia, Indonesia, Filipinas, Samoa,
Tonga, Nueva Caledonia, Islas Guam, Estados Unidos (California, New Jersey, Hawaii, Kentucky, Massachusetts), Reino Unido e Israel (Özdemir y Günal, 2012). Dentro de los principales países que compran esté producto, se encuentran: Holanda, Alemania, Francia, Inglaterra, España, Bélgica, Suiza, Canadá, Estados Unidos, Reino Unido, Italia y Brasil (FAO, 2006), además Rusia, Turquía y Japón.

En Colombia, el desarrollo de la tecnología se ha hecho, fundamentalmente, sobre el conocimiento empírico de los agricultores y trabajos de investigación en las Universidades y Corpoica. En el año 2011, el área de producción fue de 743 ha, con una producción total de $10.771 \mathrm{t}$ y un promedio de 14,5 t/ha, y sus mayores áreas en los departamentos de Boyacá (388 ha), Antioquia (154 ha) y Cundinamarca (75 ha) (Agronet, 2013). Toda la información sobre la Uchuva en Colombia se puede extraer del presente artículo.

En Ecuador la producción de Uchuva (o uvilla) se realiza por pequeños y medianos productores de la Sierra Norte entre los 2.000 a $3.000 \mathrm{msnm}$, especialmente en la provincia de Imbaburra, en donde, mediante sistemas de producción semi-tecnificado, se encuentra el 40\% de los cultivos, con producciones entre 13,6 t ha-1 en 350 ha (ALTAMIRANO, 2010). Se destaca que bajo las condiciones agroecológicas de la zona productora, por presentar periodos prolongados de luminosidad característico de la zona ecuatorial y por su temperatura estable a lo largo del año, expresa mayor coloración, contenidos de azúcares, comparada con la procedente de Kenia y Sudáfrica (Muñoz, 2003) y almidones que hacen que el fruto sea menos ácido. Brito (2002) menciona que en Quito cuando la temperatura media es de $25^{\circ} \mathrm{C}$ a los 190 días se inicia la cosecha.

En el año 2012 Ecuador exportó 104,7 t, por un valor FOB de US\$ 407.640, con precio de US\$ 3,57/kg (Banco central del Ecuador, 2012), los principales países de destino en orden de importancia de consumo son: Holanda con el 43\% del total, seguido por España, Alemania, Canadá, Reino Unido, Suecia, Bélgica, Suiza e Italia (ALTAMIRANO, 2010).

En Perú, la Uchuva se conoce como Aguaymanto, uva de monte, capulí o tomate silvestre (Angulo, 2005). El país es considerado como un importante centro de biodiversidad mundial y la cordillera de los andes peruanos ha contribuido a la gran diversidad de ecotipos de aguaymanto, base genética de los cultivos comerciales del mundo. Sin embargo, en el Perú, el cultivo en forma comercial es reciente. Se está cultivando en algunas zonas, 
como en Cajamarca, Cuzco, Huancayo, La Libertad y Ancash, especialmente para abastecer el mercado interno, y especialmente para el procesamiento con miras a la exportación (PDRS/GIZ, 2011).

A pesar de que este país es considerado como el lugar de origen, la producción es baja $\left(7\right.$ tha $\left.^{-1}\right)$. Las exportaciones, han venido en constante aumento, en 2006 se exportaron $5 \mathrm{t}$ con un valor de US\$22,817.05 (Ampex, 2008) y para 2010, las exportaciones alcanzaron 15,35 t con valor de US\$148,296.55, principalmente como fruto deshidratado. Los principales países compradores son estados Unidos con participación de 53\%, seguido por Reino Unido, Alemania, Canadá y Francia (PDRS/GIZ, 2011).

En Chile, la Uchuva es conocida como amor en bolsa o capulí (Altamirano, 2010). En este país, el cultivo cuenta con pocas referencias. Es así como para el año 2010, se contaba con alrededor de 5,5 ha cultivadas, concentrándose la producción en las regiones IV, V, VI y VII (PROCHILE, 2011). La siembra se realiza en el mes de julio, la floración se logra a los 105 días después de emergencia y la maduración comercial del fruto se alcanza en enero, a los 190 días, extendiéndose las cosechas en forma continua hasta los 290 días en el mes de marzo, en donde mueren las plantas por presencia de heladas (Agroindustria Peralillo, SF). Se estima que el rendimiento promedio nacional de $P$. peruviana es de $6 \mathrm{t} \mathrm{ha}^{-1}$. Las exportaciones de este producto son muy pocas, siendo Japón el principal país comprador, en donde alcanza un precio promedio de US\$FOB $5,66 / \mathrm{kg}$. A partir de diciembre de 2011 se autorizó el ingreso, por parte de APHIS (Animal and Plant Health Inspection Service) de frutos de uchuva a Estados Unidos (HORTIFRUT, 2013).

En Brasil, se conoce con los nombres de: Mapati, Cucura, Imbauba Mansa, Puruma o Physalis (Altamirano, 2010). Las primeras investigaciones, se iniciaron en 1999, en la estación experimental de Santa Luzia, localizada en San Paulo, convirtiéndose en los pioneros en este cultivo (CHAVES, 2006). Se reportan producciones entre 2 a 3 t de fruto en el año, que son comercializadas en mercados sofisticados de la capital paulista y en Rio de Janeiro con valores que oscilan entre US\$12 a 16 por $\mathrm{kg}$ de fruta, convirtiéndose en una excelente oportunidad para los productores rurales del centrosur de Brasil, en ciudades que tienen condiciones agroclimáticas para el cultivo, como Áurea, Roca Sales, Vacaria y Carazinho y en la región de la Sierra Catarícense, especialmente en la ciudad de Lages y Fraiburgo (RUFATO et al., 2008; ANDRADE, 2008). Actualmente, se realizan investigaciones por las Universidades Federal de Pelotas y Estatal de Santa
Catarina, en busca de potencializar a Brasil como un exportador de Physalis (MUNIZ, 2012).

En el municipio do Capão do Leão, con temperatura de $17,9^{\circ} \mathrm{C}$, en 2008 se realizó investigación para determinar la fenología de la uchuva, encontrándose que la cosecha se inicia entre 131 a 164 días después de emergencia y acumula 1.313,16 grados calor de crecimiento (LIMA, 2009).

\section{Variedades y ecotipos}

En la Uchuva hay pocas variedades y más bien genotipos que se han seleccionado en los diferentes países y que se adaptan también a los diferentes climas de las regiones específicas (ecotipos). Las introducciones a Colombia como 'Sudáfrica' y 'Kenia' tienen frutos más grandes (Fischer, 1995), debido a un mayor número de cromosomas ('Kenia' $2 \mathrm{n}=48$ vs. 'Colombia' $2 \mathrm{n}=32$ ) (Rodríguez y Bueno, 2006), pero estas dos africanas tienen concentraciones de sólidos solubles totales ( ${ }^{\circ}$ Brix) y ácido cítrico menores. Existen numerosas accesiones del género Physalis en Colombia en los bancos de germoplasma de Corpoica y de varias universidades. En América Latina y el Caribe, según Ligarreto et al. (2005) se establecieron colecciones en Brasil, Chile, Costa Rica, Ecuador, Guadelupe, Guatemala, México y el Perú. En estas regiones se encuentran variedades tradicionales y silvestres con mucha variabilidad genética. Por ejemplo, Herrera et al. (2011), en 54 accesiones encontradas en el nororiente de Colombia, determinaron un mayor contenido de ${ }^{\circ}$ Brix, pero también un rajado del fruto más alto, en materiales asilvestrados, mientras los cultivados se destacaron por un mayor rendimiento.

De Australia se reportan cultivares como 'Golden Nugget' o 'New Sugar Giant' y de Estados Unidos 'Peace', 'Giant Groundcherry', 'Goldenberry', 'Giallo Grosso', 'Giant', 'Giant Poha Berry', 'Golden Berry’ y 'Golden Berry-Long Ashton’ (FISCHER, 2000).

\section{Ecofisiología}

En los países andinos, plantaciones de uchuva se encuentran entre 1.500 y $3.000 \mathrm{msnm}$ (FAO, 1982), mientras en Colombia en altitudes entre 1.800 y $2.800 \mathrm{msnm}$. Por la luz ultravioleta más alta, en las zonas altas, la Uchuva presenta tallos principales más cortos, con hojas más pequeñas y gruesas y, por las bajas temperaturas, el primer pico de producción se aplaza comparado con cultivos en zonas más bajas (FISCHER Y MIRANDA, 2012).

Para su crecimiento y producción las 
temperaturas medias óptimas están entre 13 y $16^{\circ} \mathrm{C}$, sin embargo, Salazar et al. (2008) encontraron que la temperatura fisiológica base para el cultivo (inserción de nuevo nudos en el tallo) es de $6,29^{\circ} \mathrm{C}$. Temperaturas muy bajas, cerca de $0^{\circ} \mathrm{C}$ o por debajo queman la planta.

La Uchuva crece demasiado exuberante si hay condiciones de una intensidad lumínica reducida (por ejemplo bajo invernadero), es decir la planta se desarrolla mejor en plena luz (Fischer y Melgarejo, 2013). Mora et al. (2006) mencionan que 1.500 a 2.000 horas de luz solar directa al año son óptimas para el tamaño, la calidad y la maduración del fruto de uchuva.

No obstante, la producción de Uchuva bajo invernadero, en Chía (Cundinamarca), ha mostrado un aumento en la producción de frutos debido a un mayor desarrollo longitudinal de ramas y por consiguiente un alto índice de área foliar (IAF de 8), comparado con un IAF de solo 2 a 4 en campo abierto. Heinze y Midasch (1991) clasifican la Uchuva como una planta cuantitativa de día corto debido a que longitudes de días cortos ( 8 horas luz, comparado con 16 horas) fomentan el inicio de la floración.

Debido a su crecimiento indeterminado, necesita un suministro de agua constante para el desarrollo vegetativo y reproductivo de la planta, especialmente para el llenado del fruto. Por tal razón, es necesario que haya de 1.000 a 1.800 $\mathrm{mm}$ de precipitación bien distribuida durante el año, mientras los rangos óptimos de humedad relativa (HR) están entre 70\% y 80\% (FISCHER Y MIRANDA, 2012). Suelos encharcados durante más de 4 días causan la muerte del sistema radical; Aldana y García (2012) encontraron que uchuvas anegadas durante 6 y 8 días fueron muy afectadas, con los valores más bajos en altura de planta, área foliar, número de hojas, número de nudos, diámetro del tallo y pesos secos de los órganos de la planta, con síntomas muy marcados de marchitamiento, comparado con las de 0, 2 y 4 días en el agua.

El problema del rajado del fruto se presenta en épocas de alta precipitación y lluvias abundantes después de una época seca (FISCHER Y MELGAREJO, 2013). El agrietamiento se acentúa más en frutos sobre maduros en la planta y en aquellos de alto volumen y peso, que muchas veces coinciden con los primeros frutos del primer ciclo de cosecha (Gordillo et al., 2004) por lo que, en estos casos, se recomienda eliminar las primeras flores de la planta. Un nivel de humedad constante en el suelo (un poco por debajo de la capacidad de campo) y un nivel óptimo de calcio, boro y magnesio en la fertilización son importantes para contrarrestar esta adversidad; además, evitar sobre concentraciones de nitrógeno y eliminar las primeras flores cuando inicia la fase reproductiva, en variedades susceptibles (Fischer, 2005).

Por sus perforaciones del cáliz y las hojas la granizada la afecta notoriamente y la planta debe ser recuperada a través de poda y aplicaciones con fungicidas, mientras frutos con impactos de los granizos no son comerciables. Los vientos fuertes disminuyen su crecimiento deshidratan el suelo y las plantas por lo cual barreras naturales como otros frutales sembradas más densas (como por ejemplo el peral o la feijoa (guabeira...), o acacias, cipreses o sauces, entre otros, son recomendadas (FISCHER Y MIRANDA, 2012).

Angulo (2011) recomienda suelos sueltos con una estructura granular y una textura franco-arenoso o -arcilloso, enriquecidos con aplicaciones de materia orgánica (MO) para un pH entre 5,5 y 6,5. Además, niveles freáticos mayores de $1 \mathrm{~m}$ son óptimos para el cultivo.

\section{Propagación}

La propagación por semilla favorece el cultivo de la Uchuva, debido a su alto porcentaje de germinación (85-90\%), y estas plantas, comparado con la propagación por estacas, muestran un buen anclaje y mayor longevidad de cultivo, así mismo frutos de buena calidad y menos rajado (Klinac, 1986). Los frutos de buen tamaño son obtenidos de plantas sanas y buena productividad que se licuan a baja velocidad (Almanza, 2000) para ponerlas a fermentar durante 48 horas en un recipiente de vidrio (Angulo, 2005). Estas semillas se lavan con agua limpia y se secan en la sombra sobre un papel absorbente en un lugar fresco y ventilado (Angulo, 2011).

Debido a que enfermedades causadas por hongos como: Cladosporium sp., Phoma sp., Alternaria sp., Phytium sp., Botrytis sp. y Colletrotrichum sp. pueden infectar las semillas, se deben desinfectar con fungicidas o con hongos antagónicos (Trichoderma lingnorum o T. harzianum) (Angulo, 2005).

Para las semillas que se siembran en surcos separados entre sí por $5,0 \mathrm{~cm}$ y con $0,5 \mathrm{~cm}$ de profundidad en bandejas o en una cama de semillero que se cubre con una capa de tierra fina de $0,5 \mathrm{~cm}$, recomienda Miranda (2005) sustratos en mezcla como MO y arena lavada en diferentes proporciones $(3: 1: 1,2: 1: 1$ y $1: 1: 1)$ o sustratos como turba negra y rubia, cascarilla de arroz quemada y enriquecidos 
con micorrizas (MVA). La desinfección del sustrato es indispensable.

Después de unos 10 a 15 días las plántulas emergen y entre 25 y 30 días después de la siembra, cuando están listas para trasplantar a bolsas de color negro, perforadas y de calibre 2 , con capacidad de 1 libra (MIRANDA, 2005). Aproximadamente 60 días después de la siembra, que corresponde a una altura entre 15 y $20 \mathrm{~cm}$, se trasplantan a su sitio en campo (ANGULO, 2005).

La Uchuva responde bien a una propagación asexual mediante estacas y esquejes, in vitro, e injertos (púa terminal); sin embargo, estos métodos pueden resultar más costosos $\mathrm{y}$, en muchos casos las plantas presentan problemas como un sistema radical débil, susceptibilidad al viento y/o un periodo vegetativo reducido (FISCHER Y MIRANDA, 2012). Con esquejes (de dos nudos), provenientes de la parte apical de ramas reproductivas y sembradas en cabinas de nebulización, López et al. (2008) observaron altos porcentajes de enraizamiento, usando como sustrato arena lavada. Almanza (2000) reporta que la utilización de hormonas sintéticas (ácido indolacético [AIA], 250-1.000 mg/L o ácido indolbutírico [AIB], $50-250 \mathrm{mg} / \mathrm{L}$ ) fomenta el enraizamiento de estacas.

\section{Sistemas de cultivo y tutorado}

De acuerdo con Muniz (2012) los resultados de investigación a disposición para los cultivadores de uchuva en fincas de pequeño o mediano tamaño, son escasos con respecto al manejo del cultivo, la productividad del cultivo que permiten tomar decisiones importantes para el cultivador.

Como regla general, la Uchuva se puede sembrar en hoyos de 40 x 40 x $40 \mathrm{~cm}$ (ZAPATA et al., 2002), mientras Angulo (2011) para suelos sueltos recomienda estas dimensiones entre 20 y $30 \mathrm{~cm}$ y para suelos pesados entre 50 y $60 \mathrm{~cm}$. Al sustrato del hoyo se adicionan 2 a $4 \mathrm{~kg}$ fertilizantes orgánicos, completamente descompuestos, además químicos y correctivos, según el resultado del análisis de suelo. Para la siembra es recomendable realizarla en días lluviosos o, por lo menos, con un cielo nublado para que las plantas no se deshidraten, por lo que es necesario también garantizar un riego evitando la deshidratación de las plantas y el suelo (FISCHER Y MIRANDA, 2012). Si es necesario, se aplica cal para elevar el pH del suelo, 1 mes antes de la plantación.

Las densidades de plantación varían según el sitio agroecológico (como por ejemplo la topografía), del ecotipo (variedad) y del manejo del cultivo. Fischer y Miranda (2012), por razones de la fitosanidad y del manejo, recomiendas distancias entre plantas e hileras de 2 a $3 \mathrm{~m}$, para una densidad de 1.660 plantas/ha sembrando de $3 \mathrm{~m}$ (entre hileras) x $2 \mathrm{~m}$ (entre plantas). Angulo (2011) informa que las distancias de siembra más utilizadas en Colombia son de 2,5 x 2,5 m hasta $3 \times 3 \mathrm{~m}$, siempre teniendo en cuenta que en sitios con una mayor humedad las distancias altas favorecen la sanidad del cultivo. Así mismo, en las plantaciones en sitios pendientes Fischer y Miranda (2012) recomiendan el trazado en tresbolillo o a curvas de nivel para facilitar los manejos.

El cultivo de la Uchuva es posible intercalarlo (//) como se ha observado en las diferentes zonas productoras en Colombia: Uchuva//tomate de árbol, Uchuva//especies de hortalizas, Uchuva//arveja, Uchuva//arveja//papa (MIRANDA, 2005).

Debido al hábito arbustivo de la Uchuva, la aireación del cultivo y su apropiado manejo exige un sistema de tutorado (ANGULO, 2011). Existieron muchos diferentes tipos de soporte que pasaron por sistemas en $\mathrm{V}$ bajo, alto, triple $\mathrm{T}$, hasta uno más sencillo que ahora es usado en la mayoría de las fincas y que consiste en el amarre de las ramas laterales productivas con hilaza gruesa a dos alambres galvanizados (calibre 12 o 14) distanciados por $1 \mathrm{~m}$ y ubicados a una altura de $1,8 \mathrm{~m}$ a $2,0 \mathrm{~m}$ (Fischer y Miranda, 2012). Los postes de soporte están puestos en los extremos de cada surco, seguidos por varas cada 3 o $4 \mathrm{~m}$ dentro de la hilera (Angulo, 2011). Los cultivadores han utilizado este sistema debido a la posible asociación con arveja o frijol (ANGULO, 2005).

Para un mejor aprovechamiento de la luz favoreciendo la fotosíntesis y por consiguiente una mayor productividad y calidad de los frutos Angulo (2011) sugiere el sistema de colgado en $\mathrm{V}$ alto que consiste en enterrar dos postes de 2,5 $\mathrm{m}$ de largo 0,5 $\mathrm{m}$ en el suelo en forma de V, cada 10 a $12 \mathrm{~m}$. En los costados de la fila se colocan entre 3 y 4 alambres número 14 , ubicados cada $40 \mathrm{~cm}$, sobre los cuales se "acuestan" las ramas productivas de la planta, sin embargo Angulo (2011) no recomienda este sistema en sitios muy ventosos por la fricción del alambre con las ramas.

En Pelotas, RS, Lima et al. (2010) encontraron que el sistema de conducción en $\mathrm{V}$ invertido y el en triángulo mejoran la productividad del cultivo de uchuva, comparado con la espaldera vertical.

\section{Poda}

Siendo la Uchuva una planta con crecimiento indeterminado, como la mayoría de las especies solanáceas, el crecimiento de ramas y de los 
frutos compiten por los fotosasimilados (Fischer et al., 2011), la poda está muy relacionada con la producción y depende mucho del número y estado fitosanitario de las ramas productivas (CASIERRAPOSADA Y FISCHER, 2012).

Con respecto a la poda de formación, en la mayoría de las fincas en Colombia y, especialmente por el cambio climático con al aumento de la precipitación, se eliminan los chupones que se produjeron en la base del tallo principal, permitiendo la bifurcación apical natural del tallo vegetativo y el desarrollo de unas cuatro ramas generativas principales con sus respectivas ramas reproductivas laterales. También, en buenas condiciones de crecimiento, es posible, dejar dos a tres y máximo cuatro tallos basales vegetativos. Criollo et al. (2013) mantuvieron en tres o cuatro tallos (ramas primarias) cuatro u ocho ramas secundarios lo que aumentó el rendimiento por planta sustantivamente (Tabla 1), encontrándose diferencias entre genotipos y poca variación en el peso fresco único del fruto (entre $5,89$ y $6,16 \mathrm{~g})$.

Promedios con letras distintas indican diferencia significativa según la prueba de Tukey $(\mathrm{P} \leq 0,05) .{ }^{1}$ Letras mayúsculas para comparaciones en sentido vertical (entre genotipos); ${ }^{2}$ Letras minúsculas para comparaciones en sentido horizontal (entre número de ramas).

La poda de formación que consiste en el despunte del tallo principal, antes de la bifurcación natural que ocurre entre 8-12 nudos (Fischer, 2000), entre unos 30 a 45 días después del trasplante para inducir el crecimiento de varias ramas laterales basales, recomienda Angulo (2011) solamente para zonas que no presenten alta humedad relativa.

En la poda de mantenimiento y sanitaria se eliminan todas las ramas secas que ya produjeron y son improductivas y los chupones basales, así mismo las hojas y cálices enfermos o atacados por insectos plaga (Fischer y Miranda, 2012). Teniendo en cuenta que esta poda mejora el estado fitosanitario, incrementando la entrada de luz y aire, y favorece la producción en las ramas nuevas de la planta, debe realizarse, según Angulo (2011), cada 45 a 60 días.

En cultivos de buen estado fitosanitario, pero en el caso de un descenso de la producción y tamaño del fruto, debido a la edad de las ramas vegetativas y de los ejes productivos principales, la poda de renovación es recomendable, que se puede efectuar entre unos 18 y 24 meses desde la plantación (Fischer y Miranda, 2012). Es para tener en cuenta que esta práctica es condicionada y requiere, aparte de un óptimo estado fitosanitario de la planta y especialmente del sistema radical, una nutrición adecuada. En Colombia se mantuvieron plantas así hasta 4 años bajo invernadero con fertirrigación. De acuerdo con Fischer y Miranda (2012) el soqueo se debe realizar a unos 15 a $20 \mathrm{~cm}$ desde la base de la planta, cicatrizando los cortes formando así en pocas semanas una nueva copa.

\section{-Manejo de fertilización y agua}

Los aportes nutricionales se basan en un análisis de suelos y el estado de desarrollo de la planta. Angulo (2011) se recomienda, al momento de la siembra, una aplicación de 2 a $4 \mathrm{~kg}$ de materia orgánica por planta, usando compost o gallinaza descompuesta (tabla 2) descompuesta, y en el primer mes, después de la siembra aplicar un fertilizante compuesto (10-30-10 o 13-26-6 N-P-K; $150 \mathrm{~g} /$ planta) y en el cuarto mes $100 \mathrm{~g} /$ planta. En el estado de prefloración es importante aplicar un fertilizante que contenga alto contenido de fósforo y a partir del cuajado el potasio es indispensable para la formación de un buen tamaño de los frutos (Fischer y Miranda, 2012).

Las aplicaciones de elementos menores, recomienda Angulo (2011) cada 5 o 6 meses (30 $\mathrm{g} /$ planta), para mejorar el desarrollo de la planta y la calidad de los frutos. En la Uchuva, la falta de nitrógeno es el elemento que más influye en la disminución de la producción y su deficiencia se manifiesta en las hojas con un color verde pálido y venas rojizas, mientras se forma un menor número y longitud de ramas, disminuyendo la cantidad y el tamaño de los frutos (FISCHER Y ANGULO, 1999).

El rajado del fruto está influenciado por los nutrientes calcio y boro, Cooman et al. (2005) encontraron que la ausencia de uno de los dos elementos en la fertilización aumenta el agrietamiento en un $3 \%$, y la ausencia de los dos elementos en forma simultánea incrementa esta adversidad en un $6 \%$. Torres et al. (2013) reportan que plantas con deficiencia del $\mathrm{Ca}$ (muchas veces en aquellas que crecen en suelos de textura liviana) manifiestan en algunos frutos, sobre todo en la parte superior de la planta, cálices deformes que no cubren la totalidad del fruto, con manchas necróticas en su ápice, similares a los que muestra el tomate por deficiencia de calcio. Estos síntomas se pueden prevenir con aplicaciones de $\mathrm{CaO}$, según el análisis de suelo y utilizando fertilizantes de rápida disponibilidad como el nitrato de calcio.

En plantas de Uchuva creciendo en macetas de 25 L en arena lavada del río Martínez y Sarmiento (2008) encontraron marcadas reducciones del crecimiento de ramas por la deficiencia de $\mathrm{N}$ en la 
solución nutritiva, mientras el rendimiento (número y peso fresco de frutos) fue considerablemente disminuido por deficiencias de N, K y B (MARTÍNEZ et al., 2008). Los mismos autores encontraron que la falta del B en la solución nutritiva disminuyó el contenido de sólidos solubles en los frutos, y en aquellos deficientes en $\mathrm{N}$ y $\mathrm{P}$ aumentó la acidez titulable.

Las necesidades de agua de la uchuva a través del riego no han sido tan evaluadas como las demás prácticas, instalando por ejemplo un sistema de riego por goteo económico. Como informa Miranda (2005) se puede emplear líneas de goteo con diferentes especificaciones, goteros autocompensados de $4 \mathrm{~L} / \mathrm{h}$, en tiempos de riego variables y con la posibilidad de adaptar un sistema de fertirriego. Debido a que existen regiones cuyo régimen de lluvias es bimodal. Angulo (2011) recomienda aplicar riego dos a tres veces por semana en las épocas secas. Sin embargo, para evitar el rajado del fruto en genotipos susceptibles debido a humedades del suelo muy cambiantes, se sugiere la instalación de tensiómetros.

\section{Enfermedades, plagas y arvenses en cultivo}

En Colombia se han encontrado tres enfermedades, económicamente importantes, de las cuales el marchitamiento vascular (Fusarium oxysporum Schlecht) causa los daños más severos y en consecuencia la producción en la región afectada baja tanto que los productores abandonan este cultivo y se establecen en otras zonas del país para cumplir con las producciones exportables. El micelio del hongo penetra las raíces de manera directa, o entra a las raíces a través de heridas o en el punto de formación de las raíces laterales (FISCHER Y MIRANDA, 2012) por lo cual el manejo preventivo es de suma importancia como sembrando semillas y plántulas sanas, en suelos no infestados (GALINDO Y PARDO, 2010). El control químico es difícil debido a que el hongo ha desarrollado resistencias a muchos de los fungicidas comúnmente utilizados y a su capacidad de producir estructuras de resistencia, como las clamidosporas (RODRÍGUEZ et al., 2011).

El muerte descendente por Phoma sp. es muy frecuente en ambientes que presentan alta humedad y temperatura baja, manifestando síntomas sobre tallos, ramas, peciolos, cálices y frutos como lesiones de color amarillo a cobrizo (FISCHER Y MIRANDA, 2012). Su presencia es más frecuente en cultivos mal tutorados y con pobre drenaje (ANGULO, 2011). Es una de las enfermedades típicas que se pueden controlar y prevenir con un adecuado manejo de poda, como la sanitaria y una formación de plantas bien abiertas para lograr una buena circulación del aire dentro del cultivo, además de la aplicación de fungicidas de acción preventiva y curativa.

La mancha de la hoja y del capacho Cercospora physalidis Ell. Se presenta frecuentemente en áreas de menor altitud, en épocas de verano (GALINDO Y PARDO, 2010), además en climas en los que se alternan periodos cortos de lluvias y días secos (Fischer y Miranda, 2012).Los primeros síntomas en las hojas se manifiestan como pequeñas áreas necróticas, principalmente en forma angular (ANGULO, 2011), mientras que en el cáliz la mancha tiene un borde más definido y centros de color grisáceo (FISCHER Y MIRANDA, 2012). La aplicación de fungicidas preventivos, si se presentan las condiciones climáticas descritas y curativas en caso de infestación son recomendables.

Otras enfermedades, reportadas por Angulo (2011) en Colombia, pero de menor incidencia son: pudrición algodonosa (Sclerotinia sclerotiorum), "dampingo off" (Pythium sp.), Alternaria sp., antracnosis (Colletotrichum gloeosporioides), moho gris (Botrytis cinerea) y las bacterias Xanthomonas sp., Ralstonia solanacearum y diversos virus, como por ejemplo el del enrollamiento de las hojas.

Los primeros reportes de Brasil por Muniz et al. (2012) indican la presencia de enfermedades como Cercospora sp., Phoma sp., Alternaria sp. y Botrytis sp.

Dentro de las plagas, económicamente importantes en Colombia, se encuentra la coleóptera Epitrix cucumeris, llamada comúnmente pulguilla que ataca más en clima seco y se alimentan de los brotes tiernos y, en las hojas, causan perforaciones de diferente tamaño (FISCHER Y MIRANDA, 2012). Se controla preventivamente por medio de labores culturales como un buen desyerbe y la aplicación de riego, o curativamente, para poblaciones altas, con la aplicación de insecticidas selectivos.

Las larvas de la lepidóptera Heliothis sp. pueden inicialmente alimentarse de los cogollos, pero el daño más grave es perforando el fruto. El control debe estar dirigido a los primeros instares larvales por el monitoreo las plantas revisando los capachos y frutos, y culturalmente por la eliminación de las plantas huéspedes y uso de trampas tanto de luz como de feromonas (FISCHER Y MIRANDA, 2012).

Entre los ácaros Aculops lycopersici es la especie que más ataca las plantas de uchuva, especialmente en la fase productiva, cuando los ácaros atacan el capacho (cáliz), lo que presenta una coloración rojiza y un arrugamiento que afecta la calidad del fruto (Jerez, 2005). Las temperaturas elevadas y la escasa humedad relativa favorecen el 
desarrollo de la plaga. El control es preventivo, ya que las lluvias actúan como un controlador natural; por tanto las poblaciones de estas plagas se reducen en temporada de invierno (FISCHER Y MIRANDA, 2012).

De acuerdo con Plaza y Pedraza (2007), los arvenses asociados al cultivo de la uchuva se agrupan en 21 familias, especialmente la Asteraceae $(21,3 \%)$, Polygonaceae $(10,6 \%)$, las Brassicaceae y Scrophullariaceae (cada una con el $8,5 \%$ ), las Caryophillaceae, Cyperaceae y Poaceae (cada una con el 6,4\%) y la familia Fabaceae, con un 4,3\% del total de las especies, en su mayoría especies dicotiledóneas. Para su control es mantener el plateo limpio y/o con la aplicación de herbicidas en pre- $y$ pos-emergencia, mientras Angulo (2005) recomienda mantener una cobertura entre las hileras para evitar la erosión.

\section{Madurez del fruto y cosecha}

Según las condiciones agroecológicas, el desarrollo del fruto necesita entre 60 y 80 días (Fischer y Miranda, 2012) y presenta en aumento del tamaño y peso una curva sigmoidal simple (Fischer, 2000). La madurez fisiológica se alcanzó en Subia (Cundinamarca, Colombia, a $2.100 \mathrm{msnm}$ y $16,2^{\circ} \mathrm{C}$ ) a los 56 días después de la floración (Castañeda y Paredes, 2003), y siendo la uchuva un fruto climatérico que aumenta notoriamente su producción de etileno (TRINCHERO et al., 1999)

Los productores cosechan cuando el cáliz torna a un color amarillo que es simultáneamente con la coloración del fruto (figura 3). Según régimen de temperatura, la producción inicia entre 4 y 7 meses después de sembrada (FISCHER Y MIRANDA, 2012) y su monto puedes ser constante hasta algunos 10 a 12 meses y después disminuye. Se recomienda cosechar 2 a 3 veces por semana durante el pico de producción, preferiblemente en las horas frescas de la mañana evitando la lluvia, porque aumenta los costos del secado del cáliz y la incidencia de las enfermedades fungosas (GALVIS et al., 2005).

Como costos de producción en campo calcularon Fischer y Miranda (2012) unos $\$ 22.858 .000$ (pesos colombianos) para el primer año de un cultivo de 1 ha de uchuva, teniendo en cuenta $\$ 20.000$ para un jornal por día.

\section{Poscosecha y agroindustria}

En finca se hace una preselección y en las empresas comercializadoras se clasifica por tamaño y grados de madurez (que puede diferenciar según exigencias del importador). Las operadoras abren cada cáliz para garantizar que se exporten solamente frutos y cálices libres de enfermedades y plagas, lesiones y suciedad (GALVIS et al., 2005).

Por cuestiones de sanidad y exigencias del mercado se deshidratan los cálices con aire forzado, entre 18 y $24^{\circ} \mathrm{C}$, unos 6 horas o más, según su contenido de humedad, antes o después del empaque en cestas de plástico con un peso de frutos de 100$150 \mathrm{~g}$ (Galvis et al., 2005). Para la exportación se empacan los frutos en cajas de cartón de un peso de frutos de $1 \mathrm{~kg}$, por ejemplo 8 cestas de $125 \mathrm{~g}$, cada una, mientras para el mercado nacional son comunes canastillas de polietileno para $250 \mathrm{a} 500 \mathrm{~g}$ de frutos sin cáliz, con cubierta de vinipel, como describe para Colombia la norma NTC 5166 de Icontec (2004).

Los factores temperatura, humedad relativa (HR) y concentración del etileno en el cuarto frío son los factores ambientales más influyentes en el éxito del almacenamiento (Galvis et al., 2005). Para almacenar la uchuva hasta unas 5 semanas se usan temperaturas entre 4 y $10^{\circ} \mathrm{C}$ (con HR entre 80 y $90 \%$ y solamente sin cáliz puede incrementarse hasta el 95\%), sin embargo resiste temperaturas tan bajas hasta entre 1 y $2^{\circ} \mathrm{C}$. Alvarado et al. (2004), comprobando un tratamiento cuarentenario, la almacenaron por 16 días a $1,5^{\circ} \mathrm{C}$ sin ningún daño.

El uso de láminas de plástico para crear atmósferas modificadas pasivas ha mostrado buenos resultados en la conservación por tiempo y calidad del fruto, como las películas de polipropileno microperforado (López y Páez, 2002), poliésterpolietileno (García y Torres, citado en Fischer y Miranda, 2012) o polyolefin (Lanchero et al., 2007).

En la agroindustria, teniendo en cuenta que la uchuva con cáliz tiene $70 \%$ de pulpa, $23,6 \%$ de semillas y cáscara y $6,4 \%$ de cáliz, los productos son principalmente: (1) pulpas (natural o edulcorado) congeladas o pasterizadas, con y sin semillas; (2) bebidas (néctares y refrescos) con diferentes formulaciones; (3) uchuvas parcialmente deshidratadas, enteras, mediante ósmosis directa como pasabocas (simples o los recubiertos con chocolate o con otros productos); (4) conservas de uchuva en jarabes, salsas y compotas y (5) concentrados en forma de mermeladas, bocadillos o dulces (Camacho y Sanabria, 2005).

\section{Comercialización}

En el trópico, la uchuva es un fruto exportable con grandes ventajas comparativas debido a su producción y suministro permanente a los mercados internacionales. En Colombia, la uchuva ocupa 
el segundo lugar en las exportación de los frutos siendo este fruto uno de los más promisorios para el desarrollo de ciertas regiones del país (Fischer y Miranda, 2012).

Mientras que la producción para el mercado nacional todo depende del precio que se paga al productor que es en parte subsidiada por la fracción vendida a los mercados internacionales (SANABRIA, 2005).

Mientras que Estados Unidos importa frutos sin cáliz, requiriendo un tratamiento cuarentenario $\left(\leq 1,11^{\circ} \mathrm{C}\right.$ durante 14 días hasta $\leq 2,22^{\circ} \mathrm{C}$ por 18 días) para matar larvas de Ceratitis capitata (Flórez, 2005), a los países europeos se exportan con cáliz y sin tratamientos cuarentenarios, pero este mercado exige la certificación de EurepGap que también incluye el manejo de las Buenas Prácticas Agrícolas en finca.

Relacionado con la exportación de uchuva desde Colombia, Álvarez (2012) informa:
- Las temporadas más importantes para la exportación al continente europeo son los meses febrero-marzo y septiembre-diciembre, ingresando principalmente por los puertos de Rotterdam (Holanda) y Amberes (Bélgica).

- Alemania, Holanda, Suiza y Bélgica son los principales destinos de exportación de Colombia enviando principalmente la Uchuva convencional y solamente una pequeña producción de uchuva orgánica.

- La Uchuva convencional se envía por vía marítima desde los puertos Barranquilla, Santa Marta o Cartagena hacia Rotterdam con una duración del viaje de 18 días, mientras que la Uchuva orgánica puede ser enviada solamente por avión, debido a ser más perecedera. La ventaja de la uchuva orgánica es el buen precio en los mercados internacionales.

TABLA 1- Rendimiento ( $\mathrm{kg} / \mathrm{planta})$ de tres genotipos de uchuva manejados con diferentes sistemas de poda (Criollo et al., 2013).

\begin{tabular}{cccccc}
\hline \multirow{2}{*}{ Genotipo } & \multicolumn{2}{c}{ 3 ramas primarias } & \multicolumn{2}{c}{ 4 ramas primarias } & \\
\cline { 2 - 5 } & $\begin{array}{c}4 \text { ramas } \\
\text { secundarias }\end{array}$ & $\begin{array}{c}8 \text { ramas } \\
\text { secundarias }\end{array}$ & $\begin{array}{c}\text { 4 ramas } \\
\text { secundarias }\end{array}$ & $\begin{array}{c}8 \text { ramas } \\
\text { secundarias }\end{array}$ & \multirow{2}{*}{ Promedio } \\
\hline Reg. Nariño & $1,32 \mathrm{~A}^{1} \mathrm{~b}^{2}$ & $3,17 \mathrm{ABa}$ & $1,71 \mathrm{Ab}$ & $3,50 \mathrm{Ba}$ & $2,42 \mathrm{~B}$ \\
Kenia & $1,84 \mathrm{Ab}$ & $2,79 \mathrm{Bb}$ & $2,71 \mathrm{Ab}$ & $4,32 \mathrm{Ba}$ & $2,92 \mathrm{~B}$ \\
Silvania & $2,04 \mathrm{Ac}$ & $4,21 \mathrm{Ab}$ & $2,68 \mathrm{Ac}$ & $5,76 \mathrm{Aa}$ & $3,67 \mathrm{~A}$ \\
\hline Promedio & $1,73 \mathrm{c}$ & $3,39 \mathrm{~b}$ & $2,36 \mathrm{c}$ & $4,52 \mathrm{a}$ & 3,00 \\
\hline
\end{tabular}

TABLA 2-Plan de fertilización del cultivo de uchuva, basado en un análisis de suelo (Angulo, 2011).

\begin{tabular}{|c|c|c|c|c|}
\hline $\begin{array}{c}\text { Edad } \\
\text { planta } \\
\text { (meses) }\end{array}$ & $\begin{array}{c}\mathbf{1 0 - 3 0 - 1 0}(\mathbf{N}-\mathbf{P}-K) \\
\mathbf{1 3 - 2 6 - 6}(\mathbf{N}-\mathbf{P}-K) \\
\text { (g) }\end{array}$ & $\begin{array}{c}\mathbf{1 0 - 2 0 - 2 0}(\mathbf{N}-\mathbf{P}-K) \\
\mathbf{1 8 - 1 8 - 1 8 ( N - P - K )} \\
\mathbf{( g )}\end{array}$ & $\begin{array}{c}\text { Gallinaza } \\
\mathbf{( k g})\end{array}$ & $\begin{array}{c}\text { Elementos } \\
\text { menores } \\
\text { (g) }\end{array}$ \\
\hline 0 & & & 4 & \\
1 & 150 & 100 & 2 & 30 \\
4 & 100 & 250 & 3 & \\
7 & & 300 & 3 & 30 \\
10 & & 350 & 3 & 30 \\
13 & & 400 & 3 & \\
16 & & & & \\
\hline
\end{tabular}




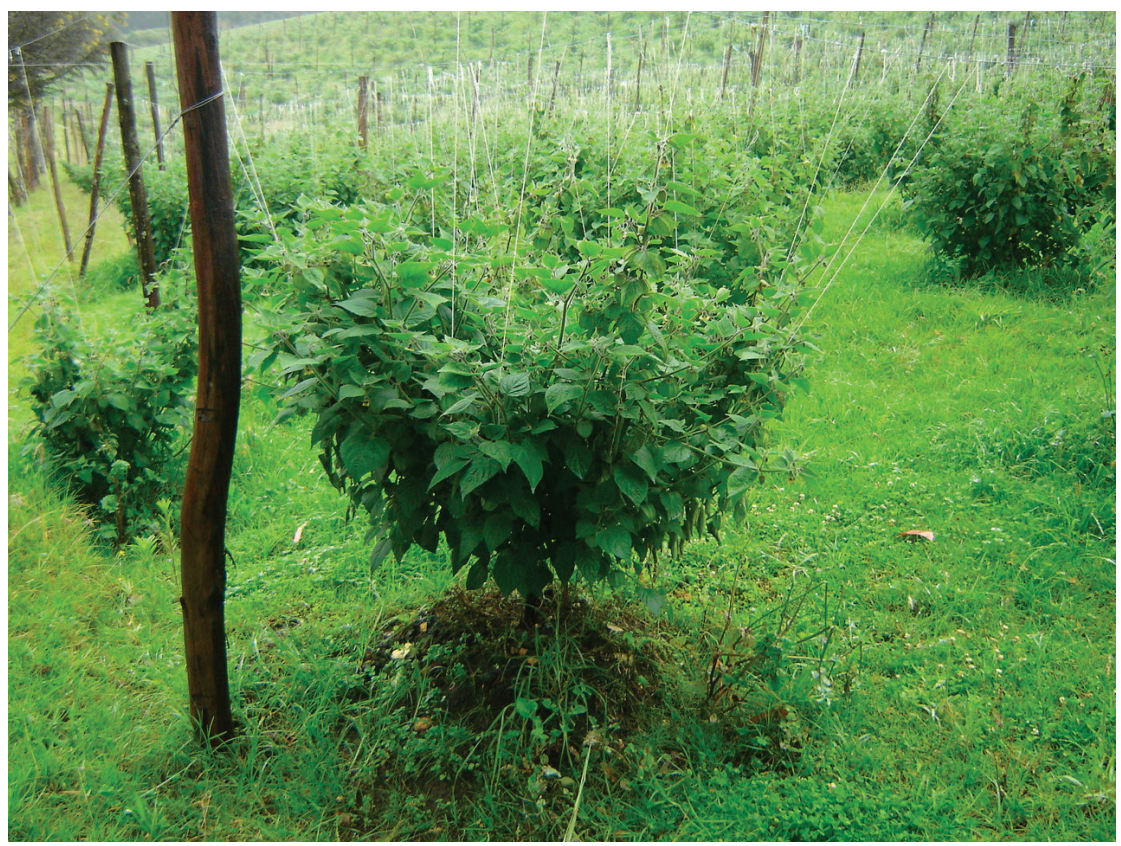

FIGURA 1- Plantación de Uchuva en Machetá, Colombia.

Foto: G. Fischer.

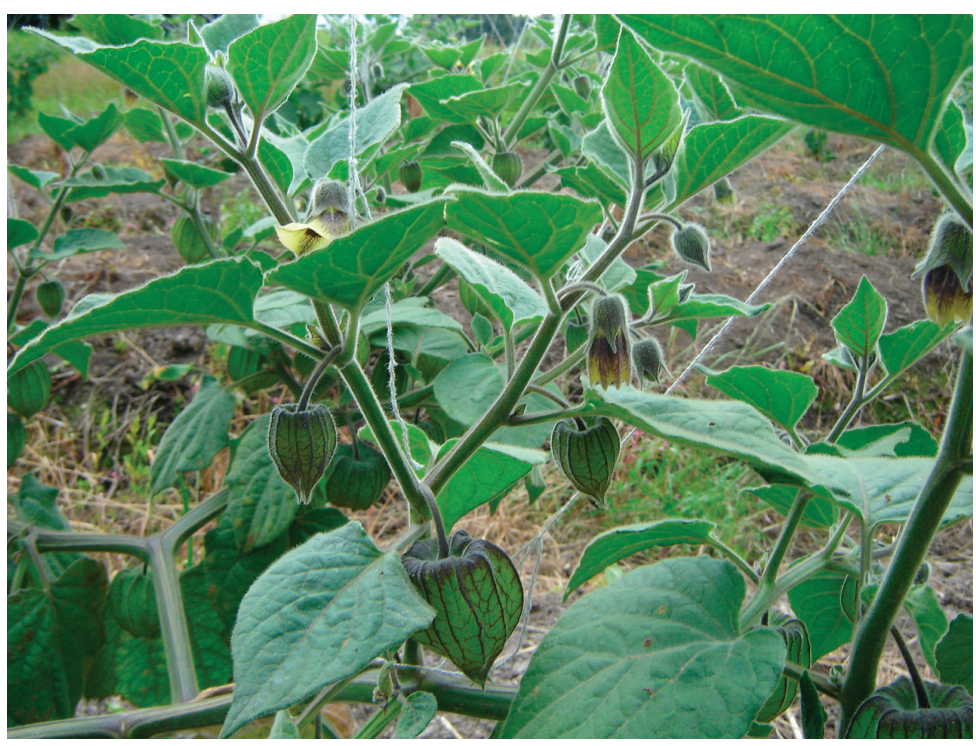

FIGURA 2- Botones florales, flores y frutos en cáliz de Uchuva.

Foto: G. Fischer. 


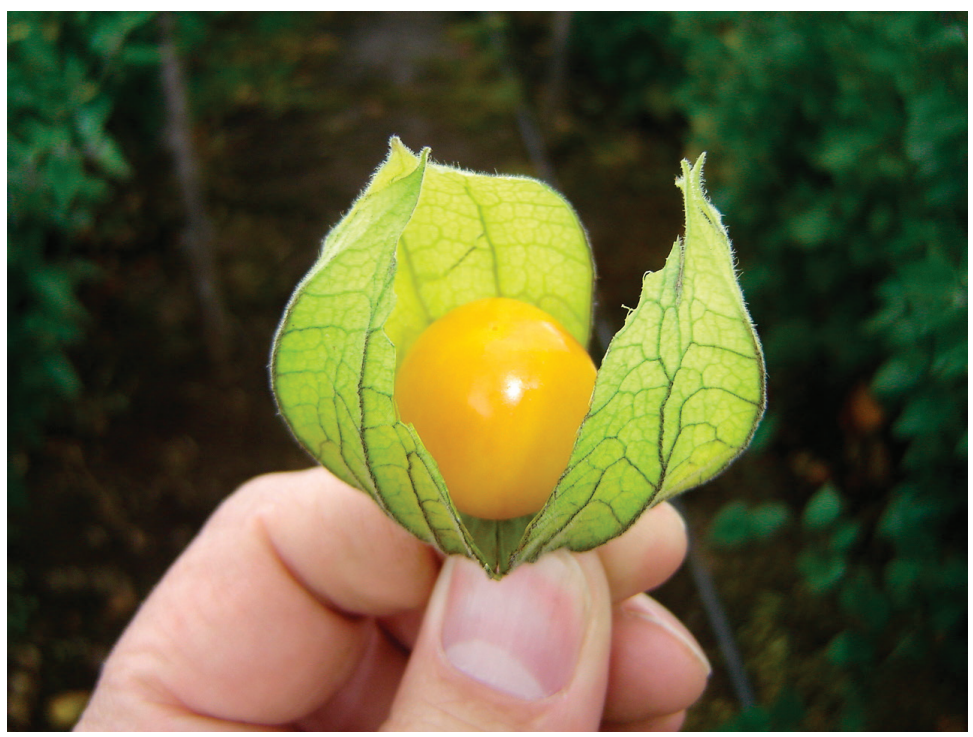

FIGURA 3- Fruto de uchuva en el punto de madurez de cosecha cuando el cáliz torna a un color amarillo Foto: G. Fischer. que es simultáneamente con la coloración del fruto.

\section{REFERENCIAS}

AGRONET. 2013. Disponível em: <http://www. agronet.gov.co/>. Acesso em: maio 2013.

ALDANA, F.; GARCÍA, P.N. Efecto del estrés por anegamiento sobre el crecimiento, desarrollo y fisiología de la uchuva (Physalis peruviana L.) bajo condiciones de invernadero. 2012. Trabajo (Grado) - Facultad de Agronomía, Universidad Nacional de Colombia, Bogotá, 2012.

ALMANZA, P.J. Propagación. En: FLÓREZ, V.J.; FISCHER, G.; SORA, A.D. (Ed.). Producción, poscosecha y exportación de la uchuva (Physalis peruviana L.). Bogotá: Universidad Nacional de Colombia, 2000. p.27-40

ALMANZA-MERCHÁN, P.J.; FISCHER, G. Fisiología del cultivo de la uchuva (Physalis peruviana L.). pp. 32-52. In: REUNIAO TÉCNICA DA CULTURA DA PHYSALIS, 2., 2012, Lages. Anais... Lages: UDESC, 2012. 128 p.

ALTAMIRANO, M. Estudio de la cadena productiva de uvilla (Physalis peruviana L.) en la Sierra norte del Ecuador. 2010. 96 f. Trabajo (Grado) - Universidad San Francisco de Quito, Colegio de Agricultura, Alimentos y Nutrición, Quito,2010.
ALVARADO, P.A.; BERDUGO. C.A.; FISCHER, G. Efecto de un tratamiento a $1,5^{\circ} \mathrm{C}$ y dos humedades relativas sobre las características físico-químicas de frutos de uchuva Physalis peruviana L. durante el posterior transporte y almacenamiento. Agronomia Colombiana, Bogotá, v.22, n.2, p.147-159, 2004.

AlVAREZ, C. Colombia: Competencia desleal y tasa de cambio afecta a los exportadores de uchuva (Physalis). 2012. Disponível em: <http://www. freshplaza.es/news_detail.asp?id $=64890>$. Acesso em: maio 2013.

AMPEX - Asociación Macroregional de Productores para Exportación. Perfil de mercado. Aguaymanto: Chiclayo-Lambayeque, 2008. 46 p.

ANDRADE, L. Physalis ou uchuva - fruta da colômbia chega ao Brasil. Revista Rural, São Paulo, v.38, p.11-12, 2008.

ANGULO, R. Uchuva Physalis peruviana. Bogotá: Bayer CropScience, 2011. 60 p.

ANGULO, R. (Ed.). Uchuva: el cultivo. Bogotá: Universidad de Bogotá Jorge Tadeo Lozano, 2005. $78 \mathrm{p}$. 
CAMACHO, G.; SANABRIA, G. Alternativas de procesamiento y transformación de la uchuva. In: FISCHER, G.; MIRANDA, D.; PIEDRAHÍTA, W.; ROMERO, J. (Ed.). Avances en cultivo, poscosecha y exportación de la uchuva (Physalis peruviana L.) en Colombia. Bogotá: Universidad Nacional de Colombia, 2005. p.191-204.

CASIERRA-POSADA, F.; FISCHER, G. La poda de frutales. In: FISCHER, G. (Ed.). Manual para el cultivo de frutales en el trópico. Bogotá: Produmedios, 2012. p.169-185.

CASTAÑEDA, G.E.; PAREDES, R.I. Estudio del proceso respiratorio, principales ácidos orgánicos, azúcares y algunos cambios físico-químicos en el desarrollo del fruto de uchuva (Physalis peruviana L.). 2003. Trabajo (Grado) - Facultad de Agronomía, Universidad Nacional de Colombia, Bogotá, 2003.

CHAVES, A. C. Propagação e avaliação fenológica de Physalis sp na região de Pelotas, RS. . 2006. 65 f. Tesis (Doctorado) - Faculdade de Agronomia "Eliseu Maciel", Universidade Federal de Pelotas, Pelotas, . 2006.

COOMAN, A.; TORRES, C.; FISCHER, F. Determinación de las causas del rajado del fruto de uchuva (Physalis peruviana L.) bajo cubierta: II. Efecto de la oferta de calcio, boro y cobre. Agronomia Colombiana, Bogotá, v.23, n.1, p.74$82,2005$.

CRFG - California Rare Fruit Growers. Cape gooseberry Physalis peruviana L. Fullerton, 1997. p.1-3.

CRIOLlO, H.; LAGOS, T.C.; FISCHER, G.; MORA, L.; ZAMUDIO, L. Efecto de la poda sobre la producción y el rajado del fruto en tres genotipos de uchuva (Physalis peruviana L.). Revista Brasileira de Fruticultura, Jaboticabal, 2013. In press.

FAO. Uchuva (Physalis peruviana L.). Fichas técnicas. 2006. Disponível em: <http://www.fao. org/inpho_archive/content/documents/vlibrary/ AE620s/Pfrescos/UCHUVA.HTM\#a2>. Acesso em: abr. 2013.

FAO. Fruit bearing forest trees technical notes. Roma, 1982. p.140-143.
FISCHER, G. El problema del rajado del fruto de uchuva y su posible control. In: FISCHER, G.; MIRANDA, D.; PIEDRAHITA. W.; ROMERO, Y J. (Ed.). Avances en cultivo, poscosecha y exportación de la uchuva (Physalis peruviana L.) en Colombia. Bogotá: Universidad Nacional de Colombia, 2005. p.55-82.

FISCHER, G. Crecimiento y desarrollo. In: FLÓREZ, V.J.; FISCHER, H.; SORA, A.D. (Ed.). Producción, poscosecha y exportación de la uchuva (Physalis peruviana L.). Bogotá: Universidad Nacional de Colombia, 2000. p.9-26.

FISCHER, G. Effect of root zone temperature and tropical altitude on the growth, development and fruit quality of cape gooseberry (Physalis peruviana L.). 1995. Tesis (Doctorado)v- HumboldtUniversität zu Berlin, Berlin, 1995.

FISCHER, G.; ANGULO, R. Los frutales de clima frío en Colombia. Ventana al Campo Andino, Bogotá, v.2, n.1, p.3-6, 1999.

FISCHER, G.; HERRERA, A.; ALMANZA, P.J. Cape gooseberry (Physalis peruviana L.) In: YAHIA, E.M. (Ed.). Postharvest biology and technology of tropical and subtropical fruits. Acai to citrus. Cambridge: Woodhead Publishing, 2011. v. 2, p.374-396.

FISCHER, G.; LÜDDERS, P.; TORRES CARVAJAL, F. Influencia de la separación del cáliz de la uchuva (Physalis peruviana L.) sobre el desarrollo del fruto. Revista Comalfi, Bogotá, v.24, n.1-2, p.3-16, 1997.

FISCHER, G.; MELGAREJO, L.M. Ecofisiología de la uchuva (Physalis peruviana L.). In: DUARTE, A.; VILLALOBOS, R.; MORENO, D.A.; GIL, Á.; FERRERES, F.; GARCÍA, C.; HEINZEN, H.; CESIO, V.; PÁSSARO, C.; OSORIO, J.; LONDOÑO, J. (Ed.). Physalis peruviana: fruta andina para el mundo: cultivo, recurso genético, agroindustria, normativa y mercado. Madrid: Editorial Académica Española, 2013.

FISCHER, G.; MIRANDA, D. Uchuva (Physalis peruviana L.). In: FISCHER, G. (Ed.). Manual para el cultivo de frutales en el trópico. Bogotá: Produmedios, 2012. p.851-873.

FLÓREZ, E. Regulación fitosanitaria para la uchuva (cape gooseberry) con destino al mercado de los Estados Unidos de América. In: FISCHER G.; 
MIRANDA, D.; PIEDRAHÍTA, W.; ROMERO, J. (Ed.). Avances en cultivo, poscosecha $\mathbf{y}$ exportación de la uchuva (Physalis peruviana L.) en Colombia. Bogotá: Universidad Nacional de Colombia, 2005. p.205-210.

GALINDO, J.R.; PARDO L.M. Uchuva (Physalis periivana $\mathbf{L}$.): producción y manejo pososcecha. Bogotá: Corredor Tecnológico Agroindustrial, Cámara de Comercio, 2010. 116 p.

GALVIS, J.A.; FISCHER, G.; GORDILLO, O.P. Cosecha y poscosecha de la uchuva. In: FISCHER, G.; MIRANDA, D.; PIEDRAHÍTA, W.; ROMERO, J. (Ed.). Avances en cultivo, poscosecha y exportación de la uchuva (Physalis peruviana L.) en Colombia. Bogotá: Universidad Nacional de Colombia,. 2005. p.165-190.

GASTELUM, D. Demanda nutrimental y manejo agronómico de Physalis peruvianum L. 2012. $74 \mathrm{f}$. Tesis (Maestría en Ciencias). -Institución de Enseñanza e Investigación en Ciencias Agrícolas, Texcoco, 2012.

GORDILLO, O.; FISCHER, G.; GUERRERO, R. Efecto del riego y de la fertilización sobre la incidencia del rajado en frutos de uchuva (Physalis peruviana L.) en la zona de Silvania (Cundinamarca). Agronomia Colombiana, Bogotá, v.22, n.1, p.5362, 2004.

GUPTA, S.K.; ROY, S.K.. The floral biology of cape gooseberry (Physalis peruviana Linn; Solanaceae, India). Indian Journal of Agricultural Science, New Delhi, v.51, n.5, p. 353-355, 1981.

HEINZE, W.; MIDASH, M. Photoperiodische Reaktion von Physalis peruviana L. Gartenbauwissenschaft, Stuttgart, v.56. n.6, p.262-264, 1991.

HERRERA, A. Manejo poscosechaEn: FLÓREZ, V.J.; FISCHER, G.; SORA, A.D. (Ed.). Producción, poscosecha y exportación de la uchuva (Physalis peruviana L.). Bogotá: Universidad Nacional de Colombia, 2000. p.109-127.

HERRERA, A.M.; ORTIZ, J.D.; FISCHER G.; CHACÓN, M.I. Behavior in production and quality of 54 cape gooseberry (Physalis peruviana L.) materials from North-eastern Colombia. Agronomia Colombiana, Bogotá, v.29, n.2, p.189-196, 2011.
HORTIFRUT. Physalis. 2013. Disponível em: $<$ http://www.hortifrut.com>. Acesso em: maio 2013.

ICONTEC- Instituto Colombiano de Normas Técnicas y Certificación. Frutas frescas. Uchuva. Especificaciones del empaque. Bogotá, 2004. (Norma Técnica Colombiana, 5166).

JEREZ, C.M. Reconocimiento de la entomofauna mayor presente en el cultivo de uchuva (Physalis peruviana L.) en el departamento de Cundinamarca y Boyacá. 2005. Trabajo (Grado) - Facultad de Agronomía, Universidad Nacional de Colombia, Bogotá, 2005.

KLINAC, D.J. Cape gooseberry (Physalis peuviana L.) production systems. New Zealand Journal of Crop and Horticultural Science, Wellington, v.14, p.425-430, 1986.

LANCHERO, O.; VELANDIA, G.; FISCHER, G;. VARELA N.C.; GARCÍA, H. Comportamiento de la uchuva (Physalis peruviana L.) en poscosecha bajo condiciones de atmósfera modificada activa. Revista Corpoica: Ciencia y Tecnología Agropecuarias, Bogotá, v.8, n.1, p.61-68, 2007.

LEGGE, A.P. Notes on the history, cultivation and uses of Physalis peruviana L. Journal of the Royal Horticultural Society, London, v.99, n.7, p.310314, 1974.

LIGARRETO, G.A.; LOBO, M.; CORREA, A. Recursos genéticos del género Physalis en Colombia. En: FISCHER, G.; MIRANDA, D.; PIEDRAHÍTA, W.; ROMERO, J. (Ed.). Avances en cultivo, poscosecha y exportación de la uchuva (Physalis peruviana L.) en Colombia. Bogotá: Universidad Nacional de Colombia, 2005. p.9-28.

LIMA, C.S. Fenologia, sistemas de tutoramento e produção de Physalis peruviana na região de Pelotas, RS. 2009. 114 f. Tesis (Maestría) - Faculdade de Agronomia Eliseu Maciel, Universidade Federal de Pelotas, 2009.

LIMA, C.S.M.; GONÇALVES, M.A.; TOMAZ, Z.F.P.; RUFATO, A.D.R.; FACHINELLO, J.C. Sistemas de tutoramento e épocas de transplante de Physalis. Ciência Rural, Santa Maria, v.40, n.12, 2472-2479, 2010. 
LÓPEZ, F.J.; GUÍO, N.R.; FISCHER, G.; MIRANDA, D. Propagación de la uchuva (Physalis peruviana $\mathrm{L}$.) mediante diferentes tipos de esquejes en distintos substratos. Revista Facultad Nacional de Agronomia, Medellín v.61, n.1, p.4347-4357, 2008.

LÓPEZ, E.; PÁEZ, L.H. Comportamiento fisiológico de la uchuva (Physalis peruviana L.) bajo condiciones de refrigeración y películas plásticas para su conservación en poscosecha. 2002. Trabajo (Grado) - Facultad de Agronomía, Universidad Nacional de Colombia, Bogotá, 2002.

MARTÍNEZ, F.E.; SARMIENTO, J. Estudio de los síntomas de deficiencia de N, P, K, Ca, Mg y $B$ en plantas de uchuva (Physalis peruviana L.). 2008. Trabajo (Grado) - Facultad de Agronomía, Universidad Nacional de Colombia, Bogotá, 2008.

MARTÍNEZ, F.E.; SARMIENTO, J.; FISCHER, G.; JIMÉNEZ, F. Efecto de la deficiencia de N, P, K, Ca, $\mathrm{Mg}$ y $\mathrm{B}$ en componentes de producción y calidad de la uchuva (Physalis peruviana L.). Agronomia Colombiana, Bogotá, v.26, n.3, p.389-398, 2008.

MIRANDA, D. Criterios para el establecimiento, los sistemas de cultivo, el tutorado y la poda de la uchuva. In: FISCHER, G.; MIRANDA, D.; PIEDRAHÍTA, W.; ROMERO, J. (Ed.). Avances en cultivo, poscosecha y exportación de la uchuva (Physalis peruviana L.) en Colombia. Bogotá: Universidad Nacional de Colombia, 2005. p.29-54.

MORA, R.; PEÑA, A.; LÓPEZ, E.; AYALA, J.J.; PONCE, D. Agrofenología de Physalis peruviana L. en invernadero y fertirriego. Revista Chapingo, Serie Horticultura, Chapingo, v.12, n.1, p.57-63, 2006.

MUNIZ, J.; KRETZSCHMAR, A.A.; RUFATO, L.; GATIBONI, L.C. Principais pesquisas realizadas com o cultivo de Physalis no Sul do Brasil. In: REUNIÃO TÉCNICA DA CULTURA DA PHYSALIS, 2., 03-04 abr. 2012, Lages. Anais... Lages: UDESC, 2012. p.56-79.

MUÑOZ, L. Análisis económico y financiero de la comercialización y producción de la uvilla como alternativa para la exportación. 2003. 89 f. Trabajo (Grado) - Escuela Superior Politécnica del Litoral, Guayaquil, 2003.
NRC - National Research Council. Goldenberry (Cape gooseberry). Washington: National Academy Press, 1989. p.241-251.

ÖZDEMIR, Y.; GÜNAL, N. Söke ovasında (Aydın) yeni bir kültür bitkisi: yer kirazı/altın çilek (Physalis peruviana). a new culture plant in söke plain (aydın): cape gooseberry/golden strawberry (Physalis peruviana). Türk Coğrafya Dergisi, Istanbul, p.35-42, 2012.

PDRS/GIZ - Proyecto de Desarrollo Rural Sostenible/GIZ Cajamarca. El aguaymanto. Cultivo promisorio de la Región Cajamarca. Diagnóstico de la cadena de valor del aguaymanto en la Región de Cajamarca. Cajamarca, 2011.96 p.

PLAZA, G.A.; PEDRAZA, M. Reconocimiento y caracterización ecológica de la flora arvense asociada al cultivo de uchuva. Agronomia Colombiana, Bogotá, v.25, n.1, p.306-313, 2007.

PROCHILE. Cultivo de Goldenberry (Physalis peruviana L.) en la zona central de Chile. 2011. Disponível em: $<$ http://www.prochile.gob.cl/noticia/ noticias-de-mercado>. Acesso em: abr. 2013.

PUENTE, L.A.; PINTO-MUÑOZ, S.A.; CASTRO, E.S.; CORTÉS, M. Physalis peruviana Linnaeus, the multiple properties of a highly functional fruit: A review. Food Research International, Essex, v.44, p.1733-1740, 2011.

REHM, S.; ESPIG, G. The cultivated plants of the tropics and subtropics. Weihersheim: Verlag Margraf, 1991.

RODRÍGUEZ, N.C.; BUENO, M.L. Estudio de la diversidad citogenética de Physalis peruviana L. (Solanaceae). Acta Biologica Colombiana, Bogotá, v.11, n.2, p.75-85, 2006.

RODRÍGUEZ, E.; PULIDO, V.; BARRERO, L.S.; GONZÁLEZ, C. El agente causal de la marchitez vascular de la uchuva. In: GONZÁLES, C.; BARRERO, L.S. (Ed.). Estudio de la marchitez vascular de la uchuva para el mejoramiento genético del cultivo. Bogotá: Cámara de Comercio de Bogotá, 2011. p.8-20. 
RUFATO, L.; RUFATO, A.R.; SCHLEMPER, C.; LIMA C.S.; KRETZSCHMAR, A.A. Aspectos técnicos da cultura da physalis. Lages: UFPel CAV/ UDESC, 2008. 100 p.

SALAZAR, M.R.; JONES, J. W.; CHAVES, B.; COOMAN A;. FISCHER G. Base temperature and simulation model for nodes appearance in cape gooseberry (Physalis peruviana L.). Revista Brasileira de Fruticultura, Jaboticabal, v.30, n.4, p.862-867, 2008..

SANABRIA, S. Situación actual de la uchuva en Colombia. In: FISCHER, G.; MIRANDA, D.; PIEDRAHÍTA, W.; ROMERO, J. (Ed.). Avances en cultivo, poscosecha y exportación de la uchuva (Physalis peruviana L.) en Colombia. Bogotá: Universidad Nacional de Colombia, 2005. p.1-8.
TORRES, C.; FISCHER, G.; MIRANDA, D. Principales fisiopatías del cultivo de uchuva (Physalis peruviana L.). In: MIRANDA, D.; CARRANZA, C.; FISCHER, G. (Ed.). Problemas de campo asociados al cultivo de uchuva (Physalis peruviana L.). Bogotá: Universidad Nacional de Colombia, 2013. p.138-146.

TRINCHERO, G.; SOIS, G.O.; CERRI, A.M.; VILELLA F.; FRANSCHINA, A. Ripening-related changes in ethylene production, respiration rate and cell-wall enzyme activity in Goldenberry (Physalis peruviana L.) a solanaceous species. Postharvest Biology and Technology, Amsterdam, v.16, p.139$145,1999$.

ZAPATA, J.L.; SALDARRIAGA, A.; LONDOÑO, M.; DÍAZ, C. Manejo del cultivo de la uchuva en Colombia. Bogotá: Corpoica, 2002. 40 p. (Boletín Técnico, 14) 